\title{
Comportamento Assintótico da Equação de Bernoulli-Euler com Dissipação Localizada e Efeito de Inércia Rotacional
}

C.R.A. da SILVA Jr., Departamento de Acadêmico de Mecânica, UTFPR, Av. Sete de Setembro, 3165, Curitiba, PR, Brasil

R.C. CHARÃO2${ }^{2}$, Departamento de Matemática, UFSC, Campus Universitário Trindade, Florianópolis, SC, Brasil.

Resumo. Neste trabalho estudamos o comportamento assintótico da energia do problema de valor inicial e de fronteira associado com a equa cão de Bernoulli-Euler com efeito de inércia rotacional e um termo não linear dissipativo localizado em uma vizinhança da fronteira do domínio. O comportamento assintótico da energia no tempo é obtido com taxas de decaimento explícitas. Esse resultado é obtido utilizando-se o lema de Nakao, estimativas de energia via multiplicadores localizados e um argumento de "compacidade-unicidade" baseado no princípio de continuação única. O comportamento assintótico é válido para a equação de Bernoulli-Euler sem efeito de inércia rotacional ou para a equa cão de placas com efeito de inércia rotacional.

\section{Introdução}

Neste trabalho estuda-se a estabilização da energia associada ao problema de valor inicial e de fronteira, para uma equação de placas do tipo Bernoulli-Euler com termo não linear dissipativo e com efeito de inércia rotacional

$$
\begin{cases}u_{t t}+\Delta^{2} u-\gamma \Delta u_{t t}-\beta\|\nabla u\|^{2} \Delta u+\rho\left(x, u_{t}\right)=0, & x \in \Omega, \quad t>0 \\ u(x, 0)=u_{0}(x), & x \in \Omega \\ u_{t}(x, 0)=u_{1}(x), & x \in \Omega \\ u(x, t)=0, & x \in \partial \Omega, t>0 \\ \frac{\partial u}{\partial n}(x, t)=0, & x \in \partial \Omega, t>0,\end{cases}
$$

com $\Omega \subset \mathbb{R}^{n}$ aberto com fronteira regular. Os dados iniciais $u_{0}$ e $u_{1}$ são tomadas tais que $u_{0} \in H_{0}^{2}(\Omega) \bigcap H^{4}(\Omega)$ e $u_{1} \in H_{0}^{2}(\Omega)$. O termo $\beta\|\nabla u\|^{2} \Delta u$ para $n=1$ descreve o problema de estabilidade elástica (flambagem) dinâmica de uma viga submetida a uma carga axial. Se $n=2$ o modelo representa uma simplificação

\footnotetext{
1 autor do trabalho

${ }^{2}$ colaborador para elaboração do trabalho
} 
do sistema completo de von Kármán que considera apenas efeitos de vibrações transversais não lineares. O efeito de inércia rotacional é representado por $\gamma \Delta u_{t t}$. O termo $\rho=\rho\left(x, u_{t}\right)$ modela fisicamente uma dissipação da energia associada a esse problema. Por exemplo, se $\rho(x, t)=a(x) u_{t}(x, t)$ então esse termo modela uma dissipação friccional linear, localizada na parte do domínio onde a função $a=a(x)$ é efetiva. No trabalho de F. Alabau-Boussouira [1] este termo dissipativo é localizado em parte da fronteira do problema. Neste trabalho a função $\rho: \Omega \times \mathbb{R} \longrightarrow \mathbb{R}$ satisfaz as seguintes condições:

i) $\rho(x, s) s \geq 0, s \in \mathbb{R}, x \in \Omega$

ii) $\rho$ e $\frac{\partial \rho}{\partial s}$ são contınuas em $\Omega \times \mathbb{R}$;

iii) Existem constantes $c_{1}, c_{2}, c_{3}, c_{4}>0$, e números $r, p \in \mathbb{R}$, com

$-1<r<\infty$ e $-1<p \leq \frac{2}{n-2}$ para $n \geq 3$ ou $-1<p<\infty$ se $n=1$

ou $n=2$, tais que :

$c_{1} a(x)|s|^{r+1} \leq|\rho(x, s)| \leq c_{2} a(x)\left[|s|^{r+1}+|s|\right], \forall s \in \mathbb{R},|s| \leq 1, \forall x \in \Omega$, $c_{3} a(x)|s|^{p+1} \leq|\rho(x, s)| \leq c_{4} a(x)\left[|s|^{p+1}+|s|\right], \forall s \in \mathbb{R},|s|>1, \forall x \in \Omega ;$

iv) $\frac{\partial \rho}{\partial s}(x, s) \geq 0, \quad \forall x \in \Omega, \forall s \in \mathbb{R}$.

sendo $a: \bar{\Omega} \rightarrow \mathbb{R}^{+}$uma função tal que $a \in L^{\infty}(\Omega)$.

A estabilização ou o estudo do comportamento assintótico da energia associada ao problema de valor inicial e de fronteira dado acima é obtida apenas para o caso em que $\gamma=0$, ou seja sem efeito de inércia rotacional, ou então no caso em que $\beta=0$. Isso é devido ao fato de não possuir um princípio de continuação única para a equação completa, ou seja para o caso em que $\gamma$ e $\beta$ são ambos não nulos. O princípio de continuação única para a equação com $\gamma=0$, é demonstrado no trabalho de Kim [4]. Para este caso são apresentados resultados sobre estabilização, ver Pazoto e Charão [2]. Para o caso $\beta=0$ o princípio de continuação única é demonstrado por Gulliver et al [3].

\section{Estabilização}

Nesta seção é apresentado um teorema contendo os principais resultados sobre a estabilização da energia para o problema de Cauchy associado à equação de BernoulliEuler apresentada em (1.1). Esse resultado é demonstrado com base no princípio da continuação única. Para equação de Bernoulli-Euler sem termo de inércia rotacional, isto é, $\gamma=0$ em (1.1), ou então para a equação (1.1) com $\beta=0$, ou seja para equação linear de placas. Para a equação completa $(\gamma, \beta>0)$ não conhecemos na literatura resultado sobre continuação única. Se tal resultado for válido, o teorema de estabilização apresentado neste trabalho será também válido para esse caso. Para localizar a dissipação, tomamos $x_{o}$ in $\mathbb{R}^{n}$ e definimos

$$
\Gamma\left(x_{o}\right)=\left\{x \in \partial \Omega:\left(x-x_{o}\right) \cdot \nu(x) \geq 0\right\},
$$

onde $\nu(x)$ denota a normal unitária exterior em $x \in \partial \Omega$. 
Agora, seja $\omega \subset \bar{\Omega}$ uma vizinhança de $\Gamma\left(x_{o}\right)$. Então além da hipótese que a função $a=a(x)$ é não negativa, também assumimos que

$$
a(x) \geq a_{o}>0, \quad \text { in } \omega .
$$

com $a_{0}$ uma constante. Desse modo a função $a=a(x)$ e portanto a dissipação representada pela função $\rho=\rho\left(x, u_{t}\right)$ na equação (1.1) é efetiva somente sobre $\omega$, isto é, a dissipação está localizada sobre $\omega$. Se $x_{0} \in \operatorname{int}(\Omega)$ então $\Gamma\left(x_{0}\right)=\Gamma$ e $\omega$ é uma vizinhança de toda fronteira de $\Omega$.

Teorema 2.1 (Estabilização). Supor que $\gamma=0$ ou $\beta=0$ e $x_{0} \in \Omega$. Então considerando-se as hipóteses feitas em (1.2) sobre as funções $a=a(x)$ e $\rho=\rho(x, s)$, tem-se que a energia associada a solução $u=u(x, t)$ do problema (1.1) tem o seguinte comportamento assintótico no tempo:

$$
E(t) \leq C(1+t)^{-\delta_{i}}, \quad i=1,2,3,4, \quad t \geq 0
$$

sendo $C$ uma constante positiva (dependendo de $E(0)$ ) e com taxa de decaimento $\delta_{i}$ definida para os seguintes casos:

Caso I: Se $r>0$ e $0<p \leq \frac{2}{n-2}$ então $\delta_{1}=\min \left\{\frac{2}{r}, \frac{8(p+1)}{4-p(n+2)}\right\}$ e $\delta_{1}=\frac{2}{r}$ se $p=0$ ou se $n=1$ ou 2 e $0 \leq p<+\infty$. Se $r=0$ e $p>0$ então $\delta_{1}=\frac{8(p+1)}{4-p(n+2)}$.

Caso II: $S$ e $r>0$ e $-1<p<0$ então $\delta_{2}=\min \left\{\frac{2}{r}, \frac{4}{p(2-n)}\right\}$ e $\delta_{2}=\frac{2}{r}$ se $n=1$ ou 2. Se $r=0$ então $\delta_{2}=\frac{4}{p(2-n)}$.

Caso III: $S e-1<r<0$ e $0<p \leq \frac{2}{n-2}$ então $\delta_{3}=\min \left\{\frac{2(r+1)}{-r}, \frac{4(p+1)}{p(n-2)}\right\} e$ $\delta_{3}=\frac{2(r+1)}{-r}$ se $p=0$ ou se $n=1$ ou 2 e $0 \leq p<+\infty$.

Caso IV: $S e-1<r<0 e-1<p<0$ então $\delta_{4}=\min \left\{\frac{2(r+1)}{-r}, \frac{4}{p(n-2)}\right\} e$ $\delta_{4}=\frac{2(r+1)}{-r}$ se $n=1$ ou 2.

Observação: Se $r=p=0$ então a energia decai exponencialmente no tempo (para a equação de Bernoulli-Euler sem o termo de inércia rotacional ver Tucsnack [7] e [8]). O teorema acima mostra o comportamento assintótico da energia associada a solução do problema definido em (1.1) e fornece as taxas expliicitas de decaimento da energia. Para demonstrar a estabilização da energia $E(t)$ deve-se mostrar que $E(t)$ satisfaz uma desigualdade do tipo:

$$
E(t)^{1+\xi_{i}} \leq C[E(t)-E(t+T)], \quad t \geq 0
$$

sendo $C$ uma constante positiva, $T>0$ está fixo e $\xi_{i}>0$ está relacionado com $\delta_{i}$ dado no teorema 2.1. Então, a propriedade de decaimento seguirá do lema de Nakao (ver Nakao [6]) 


\subsection{Identidades de Energia}

Para demonstrar o comportamento assintótico da energia torna-se necessário desenvolver algumas identidades de energia. Para isso serão úteis as seguintes funções:

Seja $h: \mathbb{R}^{n} \rightarrow \mathbb{R}^{n}$ um campo vetorial de classe $C^{2}$ tal que

$$
\begin{array}{ccc}
h(x)=\eta(x) & \text { em } & \Gamma\left(x_{0}\right) \\
h(x) \cdot \eta(x) \geq 0 & \text { em } & \Gamma \\
h(x)=0 & \text { em } & \Omega \backslash \hat{\omega}
\end{array}
$$

sendo $\hat{\omega}$ um aberto do $\mathbb{R}^{n}$ tal que $\Gamma\left(x_{0}\right) \subset \hat{\omega} \cap \bar{\Omega} \subset \omega$ (para a existência de tal campo $h$ ver Lions [5]).

Seja $m \in W^{2, \infty}(\Omega)$ uma função tal que $\frac{|\nabla m|^{2}}{m}$ e $\frac{|\Delta m|^{2}}{m}$ são limitados e

$$
\begin{array}{ccc}
0 \leq m \leq 1 & \text { em } & \Omega \\
m=1 & \text { em } & \tilde{\omega} \\
m=0 & \text { em } & \bar{\Omega} \backslash \omega
\end{array}
$$

sendo $\tilde{\omega} \subset \bar{\Omega}$ um aberto em $\bar{\Omega}$ com $\Gamma\left(x_{0}\right) \subset \tilde{\omega} \subset \omega \subset \bar{\Omega}$. Evidentemente, uma tal função $m(x)$ existe (ver Lions [5] e Tucsnak [7], [8]).

Lema 2.1 (Identidades de Energia). Sejam $h: \mathbb{R}^{n} \rightarrow \mathbb{R}^{n}$ de classe $C^{2}, m \in$ $W^{2, \infty}(\Omega), u=u(x, t)$ a solução de (1.1) e $T>0$ fixo. Então as seguintes identidades são válidas para todo $t \geq 0$ :

$$
\begin{gathered}
{\left[\int_{\Omega} u_{t} u d x\right]_{t}^{t+T}+\gamma\left[\int_{\Omega} \nabla u_{t} \cdot \nabla u d x\right]_{t}^{t+T}-\int_{t}^{t+T} \int_{\Omega}\left|u_{t}\right|^{2} d x d s} \\
+\int_{t}^{t+T} \int_{\Omega}|\Delta u|^{2} d x d s-\gamma \int_{t}^{t+T} \int_{\Omega}\left|\nabla u_{t}\right|^{2} d x d s+\beta \int_{t}^{t+T}\|\nabla u\|^{4} d s \\
+\int_{t}^{t+T} \int_{\Omega} \rho\left(x, u_{t}\right) u d x d s=0, \\
\int_{t}^{t+T} \int_{\Omega} m(x)\left[|\Delta u|^{2}-\gamma\left|\nabla u_{t}\right|^{2}-\left|u_{t}\right|^{2}+\beta\|\nabla u\|^{2}|\nabla u|^{2}+\rho\left(x, u_{t}\right) u\right] d x d s \\
=\int_{t}^{t+T} \int_{\Omega}\left(\gamma u_{t} \nabla u_{t} \cdot \nabla m-\beta\|\nabla u\|^{2} u \nabla u \cdot \nabla m\right) d x d s \\
-\gamma\left[\int_{\Omega} m(x) u \nabla u_{t} \cdot \nabla u d x\right]_{t}^{t+T}-\int_{t}^{t+T} \int_{\Omega}(u \Delta u \Delta m+2 \Delta u \nabla u \cdot \nabla m) d x d s \\
-\left\{\int_{\Omega}\left[m(x) u u_{t}+\nabla u_{t} \cdot \nabla m\right] d x\right\}_{t}^{t+T},
\end{gathered}
$$




$$
\begin{gathered}
\quad\left[\int_{\Omega} u_{t}(h \cdot \nabla u) d x\right]_{t}^{t+T}+\int_{t}^{t+T} \int_{\Omega} \rho\left(x, u_{t}\right)(h \cdot \nabla u) d x d s \\
+\frac{1}{2} \int_{t}^{t+T} \int_{\Omega}(\nabla \cdot h)\left(\left|u_{t}\right|^{2}-|\Delta u|^{2}-\beta\|\nabla u\|^{2}|\nabla u|^{2}\right) d x d s \\
+\sum_{i, j=1}^{n, n} \int_{t}^{t+T} \int_{\Omega}^{n, n}\left(2 D_{i} h_{j} D_{i} D_{j} u \Delta u+\beta\|\nabla u\|^{2} D_{i} h_{j} D_{i} u D_{j} u\right) d x d s \\
+\gamma \sum_{i, j=1}^{t+T} \int_{t}\left(D_{i} h_{j} D_{j} u D_{i} u_{t t}+h_{j} D_{i} D_{j} u D_{i} u_{t t}\right) d x d s \\
+\int_{t}^{t+T} \int_{\Omega}(\Delta h \cdot \nabla u) \Delta u d x d s=\frac{1}{2} \int_{t}^{t+T} \int_{\Gamma}(h \cdot \eta)|\Delta u|^{2} d \Gamma d s \\
{\left[\int_{\Omega} u_{t}\left(x-x_{0}\right) \cdot \nabla u d x\right]_{t}^{t+T}+\frac{n}{2} \int_{t}^{t+T} \int_{\Omega}\left(\left|u_{t}\right|^{2}-|\Delta u|^{2}-\beta\|\nabla u\|^{2}|\nabla u|^{2}\right) d x d s} \\
\int_{\Omega} \rho\left(x, u_{t}\right)\left[\left(x-x_{0}\right) \cdot \nabla u\right] d x d s+\int_{t}^{t+T} \int_{\Omega}^{n, n}\left(2|\Delta u|^{2}-\gamma\left|\nabla u_{t}\right|^{2}+\beta\|\nabla u\|^{2}|\nabla u|^{2}\right) d x d s \\
+\gamma\left(\int_{\Omega}^{t+T} \int_{t}\left(x_{t} \cdot \nabla u d x d s\right)_{t}^{t+T}+\sum_{i, j=1}^{t+T} \int_{t} \int_{\Gamma}\left(x-x_{0}\right) \cdot \eta|\Delta u|^{2} d \Gamma d s .\right. \\
=\frac{1}{2} \int_{t} \int_{j} u D_{j} u_{t t} d x d s
\end{gathered}
$$

sendo que $h^{k}$ indica a $k$-ésima componente do campo $h, D_{j}=\frac{\partial}{\partial x_{j}}, \Delta h=\left(\Delta h^{1}, \cdots\right.$, $\left.\Delta h^{n}\right), \eta=\eta(x)$ é a normal ao ponto $x \in \Gamma$ e $x_{0}$ é um ponto de $\mathbb{R}^{n}$ fixado arbitrariamente.

Demonstração. Essas identidades são obtidas utilizando-se os multiplicadores $M(u)=u, M(u)=m(x) u, M(u)=h \cdot \nabla u$ e $M(u)=\left(x-x_{0}\right) \cdot \nabla u$, respectivamente. Na identidade (2.7) não aparece o termo de fronteira da integral $\gamma \int_{t}^{t+T} \int_{\Omega} \Delta u_{t t}(h$. $\nabla u) d x d s$. Para o termo de fronteira tem-se a seguinte identidade,

$$
\int_{\Gamma} \frac{\partial u_{t t}}{\partial \eta}(h \cdot \nabla u) d \Gamma=\frac{d}{d t}\left[\int_{\Gamma} \frac{\partial u_{t}}{\partial \eta}(h \cdot \nabla u) d \Gamma\right]-\int_{\Gamma} \frac{\partial u_{t}}{\partial \eta}\left(h \cdot \nabla u_{t}\right) d \Gamma=0
$$

pois $u_{t}(t) \in H_{0}^{2}(\Omega)$ para todo $t \in[t, t+T]$. A identidade (2.8) é um caso particular da equação (2.7) quando $h(x)=x-x_{0}, \operatorname{com} x, x_{0} \in \mathbb{R}^{n}$. Em (2.6) não são usadas as propriedades definidas em (2.3) e (2.4) do campo vetorial $h$ e da função $m=m(x)$.

\subsection{Estimativas de Energia}

Nesta seção são apresentadas as estimativas necessárias para a prova do lema que será utilizado juntamente com o princıpio da continuação única para prova do teorema de Estabilização apresentado na seção. É importante observar que para todas 
as estimativas que serão realizadas a seguir, a letra $C$ poderá indicar diferentes constantes positivas. A primeira estimativa é dada pelo seguinte lema:

Lema 2.2. Existe um número $T>0$ fixo tal que a energia $E=E(t)$ associada a solução $u=u(x, t)$ do problema (1.1) satisfaz a seguinte desigualdade:

$$
\begin{gathered}
E(t) \leq C\left[E(t)-E(t+T)+\int_{t}^{t+T} \int_{\Omega}\left|\rho\left(x, u_{t}\right)\right|(|u|+|\nabla u|) d x d s\right. \\
\left.+\int_{t}^{t+T} \int_{\omega}\left|u_{t}\right|^{2} d x d s+\int_{t}^{t+T} \int_{\Omega}\left(|u|^{2}+|\nabla u|^{2}+\left|\nabla u_{t}\right|^{2}+\left|\nabla u_{t t}\right|^{2}\right) d x d s\right], \forall t>0,
\end{gathered}
$$

com $C$ uma constante positiva.

Demonstração. Para obter esta estimativa são utilizadas as desigualdades de Young e Poincaré, as propriedades da função $m=m(x)$ e a identidade de energia (2.8).

Lema 2.3. Seja $u=u(x, t)$ solução de (1.1) e $\Delta E$ definido por $\Delta E \equiv E(t)-E(t+$ $T)$. Então para $T>0$ dado no lema 2.2 e $\rho=\rho\left(x, u_{t}\right)$ satisfazendo (1.2), tem-se que

$$
\begin{gathered}
\int_{t}^{t+T} \int_{\Omega}\left|\rho\left(x, u_{t}\right)\right|[|\nabla u|+|u|] d x d s \leq C(\Delta E)^{\sigma} \sqrt{E(t)} \\
+C(\Delta E)^{\tau} E(t)^{\xi}
\end{gathered}
$$

Caso I: $r \geq 0,0 \leq p \leq \frac{2}{n-2}$ e $n \geq 3$, tem-se $\sigma=\frac{1}{r+2}, \tau=\frac{p+1}{p+2}, \xi=\frac{4+p(n+2)}{8(p+2)}$. Se $n=2$, a estimativa expressa na equação (2.9) serve para o caso $r \geq 0$ e $p \geq 0$.

Caso II: $r \geq 0,-1<p<0$ e $n \geq 2$, tem-se $\sigma=\frac{1}{r+2}, \tau=\frac{2}{4+(2-n) p}, \xi=\frac{1}{2}$.

Caso III: $-1<r<0,0 \leq p \leq \frac{2}{n-2}$ e $n \geq 3$, tem-se $\sigma=\frac{r+1}{r+2}, \tau=\frac{p+1}{p+2}$, $\xi=\frac{4-p(n-2)}{4+(p+2)}$. Se $n=2$, a estimativa é válida para o caso $-1<r<0, p \geq 0$.

Caso IV: $-1<r<0,-1<p<0$ e $n \geq 2$, tem-se $\sigma=\frac{r+1}{r+2}, \tau=\frac{2}{4+p(2-n)}$, $\xi=\frac{1}{2}$.

com $C$ uma constante positiva. Para $n=1$ as estimativas acima são as mesmas que para $n=2$.

Demonstração. Este lema é demosntrado utilzando-se as propriedades de crescimento da função $\rho$ e as desigualdade de Hölder e Poincaré.

Proposição 2.1. Seja $u=u(x, t)$ solução de (1.1) e $\Delta E$ dado por $\Delta E \equiv E(t)-$ $E(t+T)$. Para $T>0$ dado no lema 2.2 e $\rho=\rho\left(x, u_{t}\right)$ satisfazendo (1.2) então a energia associada com (1.1) satisfaz

$$
\begin{gathered}
E(t) \leq C\left[D_{i}(t)^{2}+\int_{t}^{t+T} \int_{\omega}\left|u_{t}\right|^{2} d x d s\right. \\
\left.+\int_{t}^{t+T} \int_{\Omega}\left(|u|+|\nabla u|^{2}+\left|\nabla u_{t}\right|^{2}+\left|\nabla u_{t t}\right|^{2}\right) d x d s\right], \quad \text { com } i \in\{1,2,3,4\}
\end{gathered}
$$


Caso I: $r \geq 0$ e $0 \leq p \leq \frac{2}{n-2}(0 \leq p<\infty$ se $n=2)$ tem-se $D_{1}(t)^{2}=$ $\Delta E+(\Delta E)^{\frac{2}{r+2}}+(\Delta E)^{\frac{8(p+1)}{1+p(6-n)}} ;$

Caso II: $r \geq 0$ e $0 \leq p \leq \frac{2}{n-2}(0 \leq p<\infty$ se $n=2)$ tem-se $D_{2}(t)^{2}=$ $\Delta E+(\Delta E)^{\frac{4}{3(r+2)}}+(\Delta E)^{\frac{1}{12+3 p(2-n)}}$;

Caso III: $r \geq 0 \quad e-1 \leq p<0$ tem-se $D_{3}(t)^{2}=\Delta E+(\Delta E)^{\frac{2(r+1)}{r+2}}+(\Delta E)^{\frac{8(p+1)}{12+p(6-n)}}$;

Caso IV: $-1<r<0$ e $0 \leq p \leq \frac{2}{n-2}(0 \leq p<\infty$ se $n=2)$ tem-se $D_{4}(t)^{2}=$ $\Delta E+(\Delta E)^{\frac{2(r+1)}{r+2}}+(\Delta E)^{\frac{4}{12+3 p(2-n)}}$.

Para $n=1$ as estimativas acima são as mesmas que para $n=2$.

Demonstração. Estes resultados são obtidos combinando-se os lemas (2.2) e (2.3) e a desigualdade de Young.

Proposição 2.2. Supor que $x_{0} \in \operatorname{int}(\bar{\Omega})$ e que $\gamma=0$ ou $\beta=0$. Então existe uma constante $C>0$, tal que

$$
\begin{aligned}
\int_{t}^{t+T} & \int_{\Omega}\left[|u|^{2}+|\nabla u|^{2}+\left|\nabla u_{t}\right|^{2}+\left|\nabla u_{t t}\right|^{2}\right] d x d s \\
& \leq C\left[D_{i}(t)^{2}+\int_{t}^{t+T} \int_{\omega}\left|u_{t}\right|^{2} d x d s\right]
\end{aligned}
$$

sendo $u=u(x, t)$ solução de (1.1) para dados iniciais $u_{0}$ e $u_{1}$ satisfazendo $E(0) \leq$ $R, R>0$ fixado, com $C=C(R)$ e $D_{i}=D_{i}(t)$ definido na proposição 2.1.

Demonstração. A estimativa acima é obtida por contradição utilizando-se para isso o princípio de continuação única.

\section{Demonstração do Teorema de Estabilização}

Para provar o resultado do teorema 2.1 é suficiente considerar que $u_{0} \in H_{0}^{2}(\Omega) \cap$ $H^{4}(\Omega), u_{1} \in H_{0}^{2}(\Omega)$. Das proposições 2.1 e 2.2 tem-se

$$
E(t) \leq C\left[D_{i}(t)^{2}+\int_{t}^{t+T} \int_{\omega}\left|u_{t}\right|^{2} d x d s\right]
$$

com $D_{i}(t), i=1,2,3,4$, dados no a proposição 2.1. Para derivar a estimativa de decaimento deve-se estimar o último termo do integrando de (3.1)

$$
E(t) \leq C\left[D_{i}(t)^{2}+\int_{t}^{t+T} \int_{\Omega_{1}} \frac{a(x)}{a_{0}}\left|u_{t}\right|^{2} d x d s+\int_{t}^{t+T} \int_{\Omega_{2}} \frac{a(x)}{a_{0}}\left|u_{t}\right|^{2} d x d s\right],
$$

com $D_{i}(t), i=1,2,3,4$, dados na proposição 2.3 e $\Omega_{1}=\left\{x \in \Omega|| u_{t}(x, 0) \mid \leq\right.$ $1\}, \Omega_{2}=\left\{x \in \Omega|| u_{t}(x, 0) \mid>1\right\}$. A seguir será demonstrado o Caso II do teorema 
de estabilização, os demais casos são análogos.

Caso II: $r>0$ e $-1 \leq p<0$.

$$
\int_{t}^{t+T} \int_{\omega} a(x)\left|u_{t}\right|^{2} d x d s=\int_{t}^{t+T} \int_{\Omega_{1}} a(x)\left|u_{t}\right|^{2} d x d s+\int_{t}^{t+T} \int_{\Omega_{2}} a(x)\left|u_{t}\right|^{2} d x d s .
$$

Para a primeira integral dada na equação (3.3) pode-se obter uma estimativa utilizandose a desigualdade de Hölder

$$
\int_{t}^{t+T} \int_{\omega}\left|u_{t}\right|^{2} d x d s \leq \int_{t}^{t+T} \int_{\Omega_{1}} \frac{a(x)}{a_{0}}\left|u_{t}\right|^{2} d x d s+\int_{t}^{t+T} \int_{\Omega_{2}} \frac{a(x)}{a_{0}}\left|u_{t}\right|^{2} d x d s .
$$

A integral $\int_{t}^{t+T} \int_{\Omega_{1}} a(x)\left|u_{t}\right|^{2} d x d s$ pode ser estimada utilizando-se da estimativa expressa em (3.4)

$$
\int_{t}^{t+T} \int_{\Omega_{1}} a(x)\left|u_{t}\right|^{2} d x d s \leq C(\Delta E)^{\frac{2}{r+2}}
$$

com $C>0$ dependendo de $|\Omega|, r,\|a\|_{L^{\infty}(\Omega)}$ e $c_{1}$. Para estimar a segunda integral da equação (3.4) será utilizado o fato de que $L^{\infty}\left(0, T ; H^{1}(\Omega)\right) \hookrightarrow L^{\infty}\left(0, T ; L^{\alpha}(\Omega)\right)$. Para obter $\alpha$ será utilizado resultados de imersão $m=1, p=2$ e $n \geq 3 \mathrm{mp}=1.2<$ $3 \rightarrow \alpha=\frac{2 n}{n-2}$. Desta forma tem-se que $H_{0}^{1}(\Omega) \hookrightarrow L^{\frac{2 n}{n-2}}(\Omega) \Rightarrow L^{\infty}\left(0, T ; H^{1}(\Omega)\right) \hookrightarrow$ $L^{\infty}\left(0, T ; L^{\frac{2 n}{n-2}}(\Omega)\right)$.

$$
\int_{t}^{t+T} \int_{\Omega_{2}} a(x)\left|u_{t}\right|^{2} d x d s=\int_{t}^{t+T} \int_{\Omega_{2}} a(x)\left|u_{t}\right|^{\lambda}\left|u_{t}\right|^{2-\lambda} d x d s .
$$

A desigualdade de Hölder será utilizada na equação (3.6), com $\frac{1}{l}+\frac{1}{l^{\prime}}=1$ e fazendose $l^{\prime}=\frac{p+2}{\lambda}$ e $l=\frac{2 n}{(2-\lambda)(n-2)}$.

$$
\begin{gathered}
\int_{t}^{t+T} \int_{\Omega_{2}} a(x)\left|u_{t}\right|^{\lambda}\left|u_{t}\right|^{2-\lambda} d x d s \leq \\
{\left[\int_{t}^{t+T} \int_{\Omega_{2}}\left|a(x) u_{t}^{\lambda}\right|^{l^{\prime}} d x d s\right]^{\frac{1}{l^{\prime}}}\left[\int_{t}^{t+T} \int_{\Omega_{2}}\left|u_{t}\right|^{(2-\lambda) l} d x d s\right]^{\frac{1}{l}}}
\end{gathered}
$$

Observando que $\lambda l^{\prime}=p+2$ a primeira integral de (3.7)

$$
\left[\int_{t}^{t+T} \int_{\Omega_{2}}\left|a(x) u_{t}^{\lambda}\right|^{l^{\prime}} d x d s\right]^{\frac{1}{l^{\prime}}}=\left[\int_{t}^{t+T} \int_{\Omega_{2}}|a(x)|^{l^{\prime}}\left|u_{t}\right|^{p+2} d x d s\right]^{\frac{1}{l^{\prime}}} .
$$

Notando que

$$
|a(x)|^{l^{\prime}}=|a(x)|^{\frac{p+2}{\lambda}} \frac{|a(x)|}{|a(x)|} \leq \frac{\|a\|_{L^{\infty}(\Omega)}^{p+2}}{a_{0}}|a(x)| .
$$


Substituindo-se a estimativa expressa em (3.8) na desigualadade (3.9) tem-se

$$
\left[\int_{t}^{t+T} \int_{\Omega_{2}}\left|a(x) u_{t}^{\lambda}\right|^{l^{\prime}} d x d s\right]^{\frac{1}{l^{\prime}}} \leq C\left[\int_{t}^{t+T} \int_{\Omega_{2}}|a(x)|\left|u_{t}\right|^{p+2} d x d s\right]^{\frac{1}{l^{\prime}}},
$$

a constante $C$ depende de $\|a\|_{L^{\infty}(\Omega)}, a_{0}, p, l^{\prime}$ e $\lambda$. A segunda integral de (3.8) é estimada observando-se que $(2-\lambda) l=\frac{2 n}{n-2}$ e utilizando-se o seguinte resultado de imersão com $m . p=1.2<3$ então $H_{0}^{1}\left(\Omega_{2}\right) \hookrightarrow L^{\frac{2 n}{n-2}}\left(\Omega_{2}\right)$

$$
\begin{gathered}
\left\|u_{t}(t)\right\|_{L^{\frac{2 n}{n-2}}\left(\Omega_{2}\right)} \leq C\left\|u_{t}(t)\right\|_{H_{0}^{1}\left(\Omega_{2}\right)}=\left[\int_{\Omega_{2}}\left(\left|u_{t}\right|^{2}+\left|\nabla u_{t}\right|^{2}\right) d x\right]^{\frac{1}{2}} \\
\leq\left[\int_{\Omega}\left(\left|u_{t}\right|^{2}+\left|\nabla u_{t}\right|^{2}\right) d x\right]^{\frac{1}{2}} \leq C E(t)^{\frac{1}{2}} \leq C E(0)^{\frac{1}{2}}
\end{gathered}
$$

Integrando-se a estimativa dada em (3.11) no intervalo $(t, t+T)$ tem-se

$$
\int_{t}^{t+T}\left\|u_{t}(s)\right\|_{L^{\frac{2 n}{n-2}}\left(\Omega_{2}\right)} d s \leq C T E(0)^{\frac{1}{2}} .
$$

A segunda integral da equação (3.3) é estimada como

$$
\int_{t}^{t+T} \int_{\Omega_{2}} a(x)\left|u_{t}\right|^{2} d x d s \leq C T E(0)^{\frac{1}{2}}\left[\int_{t}^{t+T} \int_{\Omega_{2}} a(x)\left|u_{t}\right|^{\lambda l^{\prime}} d x d s\right]^{\frac{1}{l^{\prime}}} .
$$

Notando que $l^{\prime}=\frac{p+2}{\lambda}$ e $\lambda=\frac{4(p+2)}{4+p(2-n)}$ então $l^{\prime}=\frac{4+p(2-n)}{4}$,

$$
\begin{gathered}
\int_{t}^{t+T} \int_{\Omega_{2}} a(x)\left|u_{t}\right|^{2} d x d s \leq C T E(0)^{\frac{1}{2}}\left[\int_{t}^{t+T} \int_{\Omega_{2}} a(x)\left|u_{t}\right|^{p+2} d x d s\right]^{\frac{4}{4+p(2-n)}} \\
\leq C\left[\int_{t}^{t+T} \int_{\Omega_{2}} \rho\left(x, u_{t}\right) u_{t} d x d s\right]^{\frac{4}{4+p(2-n)}}=C \Delta E^{\frac{4}{4+p(2-n)}}
\end{gathered}
$$

Substituindo-se as estimativas dadas em (3.5) e (3.14) na estimativa expressa em (3.2) obtém-se

$$
E(t) \leq C\left[D_{2}(t)^{2}+(\Delta E)^{\frac{2}{r+2}}+(\Delta E)^{\frac{4}{4+p(2-n)}}\right] .
$$

Para $r \leq 0,-1 \leq p \leq 0$ e $n>2$ da proposição 2.3 tem-se

$$
D_{2}(t)^{2}=\Delta E+(\Delta E)^{\frac{2}{r+2}}+(\Delta E)^{\frac{4}{4+p(2-n)}} .
$$

Colocando-se a equação (3.16) na estimativa (3.14)

$$
E(t) \leq C\left[\Delta E+(\Delta E)^{\frac{2}{r+2}}+(\Delta E)^{\frac{4}{4+p(2-n)}}\right] .
$$


Definindo-se $K_{2}=\min \left\{\frac{2}{r+2}, \frac{4}{4+p(2-n)}\right\}$ e da estimativa acima tem-se que

$$
\sup _{t \leq s \leq t+T} E(s)^{\frac{1}{K_{2}}} \leq C \Delta E .
$$

Observando-se que $\frac{1}{K_{2}}>1 \rightarrow \frac{1}{K_{2}}=1+\xi$, resolvendo-se para $\xi$ tem-se $\xi=$ $\frac{1-K_{2}}{K_{2}}$. Fazendo-se $\delta_{2}=\frac{1}{\xi}$ e aplicando-se o lema de Nakao obtém-se a prova do teorema para o Caso II com a seguinte taxa de decaimento de energia $\delta_{2}=$ $\min \left\{\frac{2}{r}, \frac{4}{p(2-n)}\right\}$.

\section{Referências}

[1] F. Alabau-Boussouira, Convexity and Weighted Integral Inequalities for Energy Decay Rates of Nonlinear Dissipative Hyperbolic Systems, Applied Mathematics and Optimization, 51, (2005), 67-91.

[2] R.C. Charão, E. Bisognin, V. Bisognin, A.F. Pazoto, Asymptotic behavior of a Bernoulli-Euler type equation with nonlinear localized damping, Contributions to Nonlinear Analysis - Progress in nonlinear partial differential equations and their applications, 66, (2005), 67-91.

[3] R. Gulliver, I. Lasiecka, W. Littman, R. Triggiani, The Case for Differential Geometry in the Control of Single and Coupled PDES: The Structural Acoustic Chamber, IMA Volumes in Mathematics and its Applications, 137, (2004), 73182.

[4] J.U. Kim, A unique continuation property of a beam equation with variable coefficients, in estimation and control of distributed parameter sustems, International Series of Numerical Mathematics, 100, (1991), 197-205.

[5] J.L. Lions, Exact controllability, stabilization and perturbations for distributed systems, SIAM Rev., 30,(1988), 1-68.

[6] M. Nakao, Decay of solutions of the wave equation with a local nonlinear dissipation, Math. Ann., 305, (1996), 403-417.

[7] M. Tucsnak, Semi-internal stabilization for a non-linear Bernoulli-Euler equation, Mathematical Methods in the Applied Sciences, 19, (1996), 897-907.

[8] M. Tucsnak, Stabilization of Bernoulli-Euler beam by means of a pointwise feedback force, SIAM J. Control Optim., 39, (2000) 1160-1181. 